\title{
Advanced Protocol to Prevent Man-in-the-middle Attack in SCADA System
}

\author{
Sangkyo Oh, Hyunji Chung, Sangjin Lee and Kyungho Lee \\ Center for Information Security Technologies, Korea University, Seoul, Korea \\ \{darkapple, foryou7187, sangjin, kevinlee\}@korea.ac.kr
}

\begin{abstract}
SCADA system is a computer system that monitors and controls the national infrastructure or industrial process including transportation facilities, water treatment and distribution, electrical power transmission and distribution, and gas pipelines. If a SCADA system is infected by a malicious worm, such as the Stuxnet, disaster is inevitable. Since the appearance of Stuxnet, researchers focused on detecting this intrusion in SCADA networks. As a result, various methods have been presented by researchers. One of them is to monitor traffic and detect anomalous patterns. However, it is not able to detecting a spoofed packet. This study present three cases of system anomaly by example of pattern based on real data of PROFINET/DCP protocol. And propose protection method using the authentication.
\end{abstract}

Keywords: SCADA system, PROFINET DCP, Authentication

\section{Introduction}

Stuxnet is the most dangerous computer worm that attacks PC's. Such danger is present because the Stuxnet targets the Siemens SCADA system, which is an industrial control system that monitors and controls industrial, infrastructure, or facility-based processes [1,2]. Ever since the emergence of Stuxnet, there has been vast research on techniques for intrusion detection in the SCADA system.

There are various methods for anomaly detection, such as an analysis of traffic pattern [3, $4,16]$, analysis of $\log$ in SCADA systems [5], model-based monitoring [6], and etc. These methods cannot help being confined to five limitations different from individual PC [7]. Firstly, it is impossible to change the existing system and software. Secondly, security control systems cannot be installed on the SCADA hardware and software systems. Thirdly, blocking network traffic is out of the question. Fourthly, shutting off or rebooting the system is, once again, impossible. Fifthly, additional network traffic should not be created.

One method to detect intrusion in the SCADA system is to monitor traffic and detect anomalous patterns. This method concentrates on whether or not the network traffic is in a normal pattern. This is also the appropriate method to detect flooding attacks, such as DOS and DDOS. However, spoofed contents of packets cannot be detected through this method. If an intelligent attacker sends spoofed contents of packets with a normal pattern, these packets can bypass detection, based on the traffic patterns.

The object of contents monitoring is PROFINET/DCP (Dynamic Configuration Protocol) packet's fields. It is possible to attack SCADA network using PROFINET/DCP because this protocol takes responsibility for device's configuration.

This study present three cases of system anomaly by example of pattern based on real data of PROFINET/DCP protocol. And propose protection method using the authentication in advanced protocol structure. 


\section{PROFINET/DCP}

PROFINET is the open industrial Ethernet standard of PROFIBUS and PROFINET International [8] and a set of fieldbus protocol [11]. There are seven PROFINET protocols, such as PROFINET/CBA, PROFINET/DCP, PROFINET/IO, PROFINET/MRP, PROFINET/MRRT, PROFINET/PTCP, PROFINET/RT [11]. PROFINET/IO and CBA are independent and other protocols are helper protocols [11].

PROFINET/DCP is a protocol that reads out and sets device parameters, such as devices' names, IP addresses and MAC addresses in PROFINET/IO systems[12, 13]. Format of PROFINET/DCP is like Figure 1 [10].

\begin{tabular}{|c|}
\hline PROFINET/DCP \\
\hline FrameID \\
\hline SeviceID \\
\hline Service Type \\
\hline Xid \\
\hline Response Delay \\
\hline DCP Data Length \\
\hline Block \\
\hline
\end{tabular}

Figure 1. PROFINET/DCP

Device Access Point supports DHCP(Dynamic Host Configuration Protocol) because of allocating the IP addresses. PROFINET/IO devices support DCP(Dynamic Configuration Protocol) for address translation[14]. DHCP and DCP can be used for the same goal.

ServiceID is identification of communication, such as Get, Set and Identify [9]. ServiceType is type of communication, such as Request, Response [9]. Xid means identification of transaction $[9,15]$. NameOfStation means source device's name.

\section{Three Cases of Attack Scenario}

Chapter 3 introduces three cases that can cause malfunction in SCADA using PROFINET/DCP's properties. Three cases assume that attacker controls devices in SCADA.

\subsection{Packet Flooding}

Using PROFINET/DCP, device in SCADA identify whether or not specific device's name exists using request packet. For example, device 1 sends request packet to other devices. "NameOfStation" field of request packet contains device2's name. At this time, if device2 is operable, device 2 sends response packet to device 1 . Using this property, attacker pretends to device 1 and sends a lot of request packets to device 2 like Figure 2. When devices of SCADA process large amount of packets, it is possible for devices to take down. Flooding attack can be fatal attack in SCADA networks. To succeed flooding attack, packets to be sent must be forged packets like real packets. This is because packets that contain strange value are dropped and attack will be unsuccessful. 


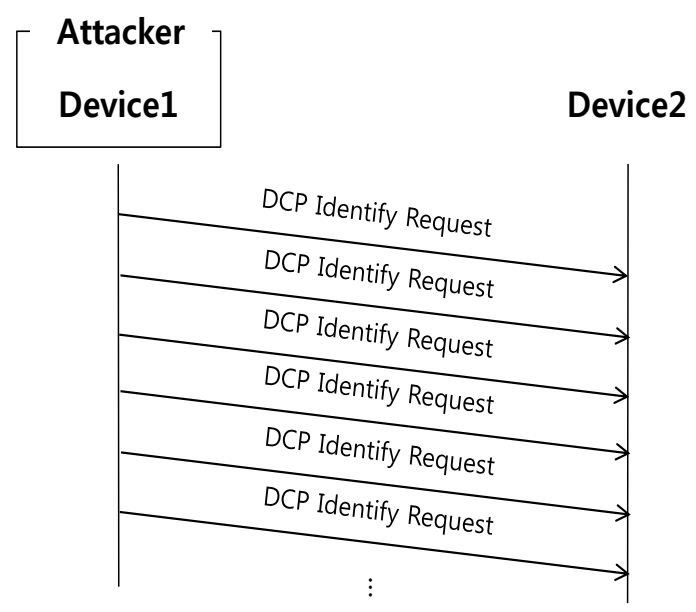

Figure 2. Process of Packet Flooding

\subsection{Packet Sniffing}

Request and response is communication between device and device. Attacker monitors communication between device and identifies devices' name. Attacker targets one device and sniffs its response packet like Figure 3. Attacker intercepts all response packets that device2 sends. As a result, other devices cannot recognize existence of device 2 that sent response packet. It means device2 doesn't work. Stopping device is risky in SCADA systems.

\section{Device1 Device2}

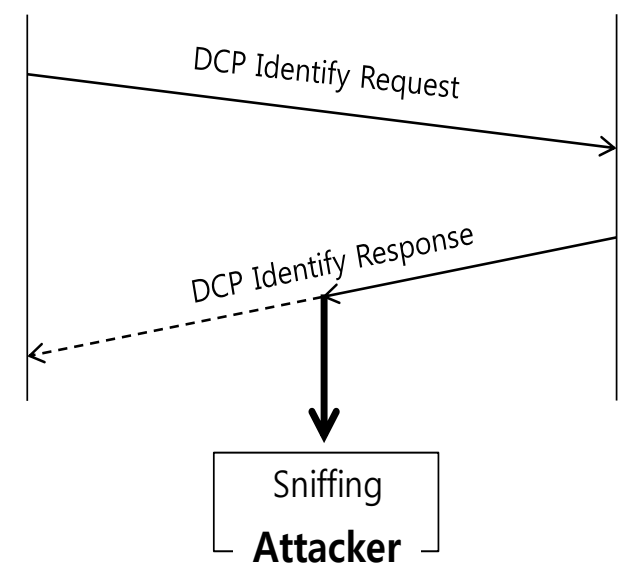

Figure 3. Process of Response Packet Sniffing

\subsection{Packet Xid Spoofing}

Device1 forges Xid of request packet like Figure 4. Xid spoofing has no malicious capability per se. However, Xid spoofing means attacker breaks into SCADA systems. It is important to detect Xid spoofing. 


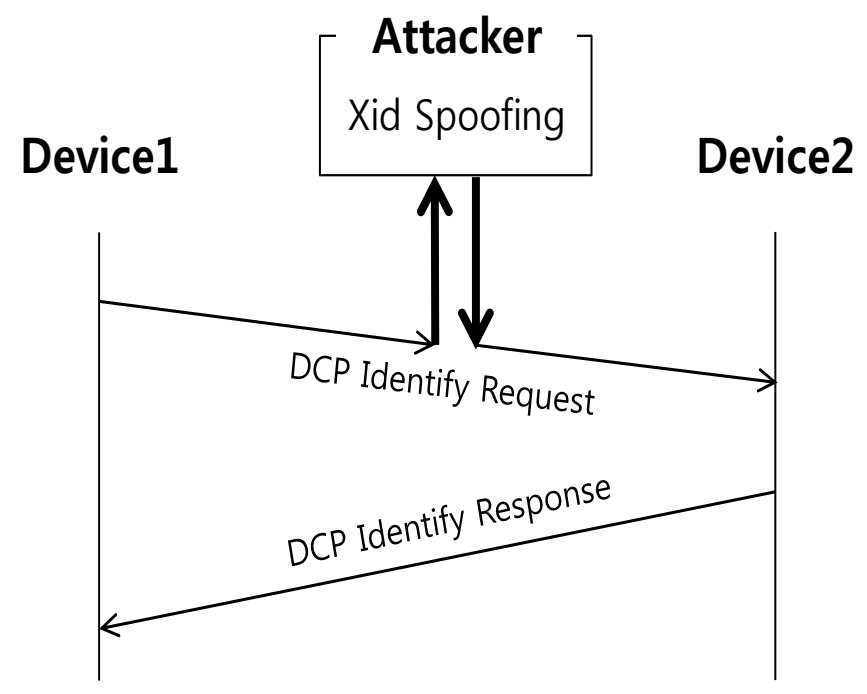

Figure 4. Process of Packet Spoofing

\section{Pattern of PROFINET/DCP Packets}

During contents monitoring in PROFINET/DCP of SCADA network, it identifies pattern of packets. Each device sends request packets to other devices according to the pattern. According to real data, pattern is like Table 1.

Table 1. One Cycle of Normal Pattern

\begin{tabular}{|c|c|c|c|c|}
\hline No. & Source & Destination & Name of Station & Number of Packets \\
\hline 1 & SiemensA_xx:xx:xx(A) & PN-MC_xx:Xx:Xx & Station A & 8 \\
\hline 2 & SiemensA_xx:xx:Xx(B) & PN-MC_xx:xx:xx & Station B & 2 \\
\hline 3 & SiemensA_xx:xx:xx(C) & PN-MC_xx:xx:xx & Station C & 2 \\
\hline 4 & SiemensA_xx:xx:xx(D) & PN-MC_xx:xx:xx & Station D & 2 \\
\hline 5 & SiemensA_xx:xx:xx(E) & PN-MC_xx:Xx:xx & Station E & $2 / 4 / 6$ \\
\hline 6 & SiemensA_xx:xx:xx(F) & PN-MC_xx:xx:xx & Station F & 2 \\
\hline 7 & SiemensA_xx:xx:xx(E) & PN-MC_xx:xx:xx & Station E & $6 / 4 / 2$ \\
\hline 8 & SiemensA_xx:xx:xx(G) & PN-MC_xx:xx:xx & Station G & 2 \\
\hline 9 & SiemensA_xx:xx:xx(H) & PN-MC_xx:Xx:Xx & Station $\mathrm{H}$ & 2 \\
\hline 10 & SiemensA_xx:xx:xx(I) & PN-MC_xx:xx:xx & Station I & 2 \\
\hline 11 & SiemensA_xx:xx:xx(H) & PN-MC_xx:xx:xx & Station $\mathrm{H}$ & 2 \\
\hline 12 & SiemensA_xx:xx:xx(I) & PN-MC_xx:xx:xx & Station I & 2 \\
\hline 13 & SiemensA_xx:xx:xx(H) & PN-MC_xx:xx:xx & Station H & 2 \\
\hline 14 & SiemensA_xx:xx:xx(I) & PN-MC_xx:Xx:Xx & Station I & 2 \\
\hline 15 & SiemensA_xx:xx:xx(H) & PN-MC_xx:xx:xx & Station H & 2 \\
\hline
\end{tabular}

This result shows same pattern is repeated periodically. It means that someone who had bad purpose can manipulate these patterns to attack the system. 


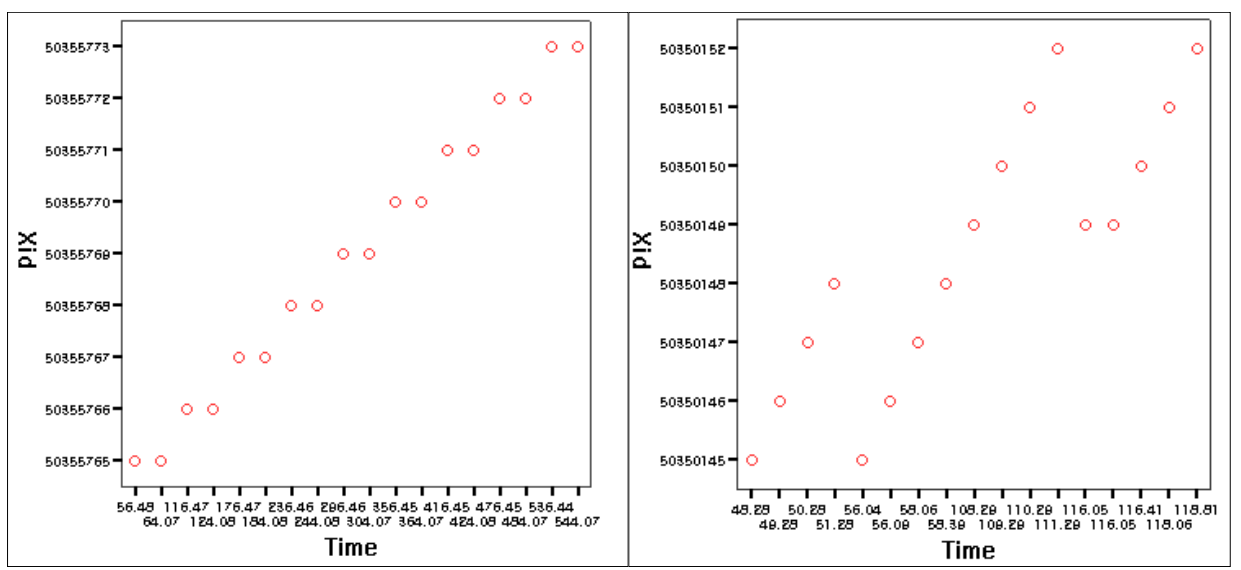

Figure 5. Change in Xid(Device1 and Device2)

The second factor is change in Xid by each device. According to real data, change in Xid by each device is different. Figure 5 shows change in Xid by device1 and device2.

As shown above, the actual traffic flow of PROFINET/DCP had some pattern, thus PROFINET/DCP protocol structure and data analysis of the packet can identify the possibility of actual attack.

\section{DNPSec}

DNPSec [17] authentication and integrity framework capabilities will verify the frame origin, assure that the frame sent is the frame received, assure that the network headers have not changed since the frame was sent, and give anti-replay protection.

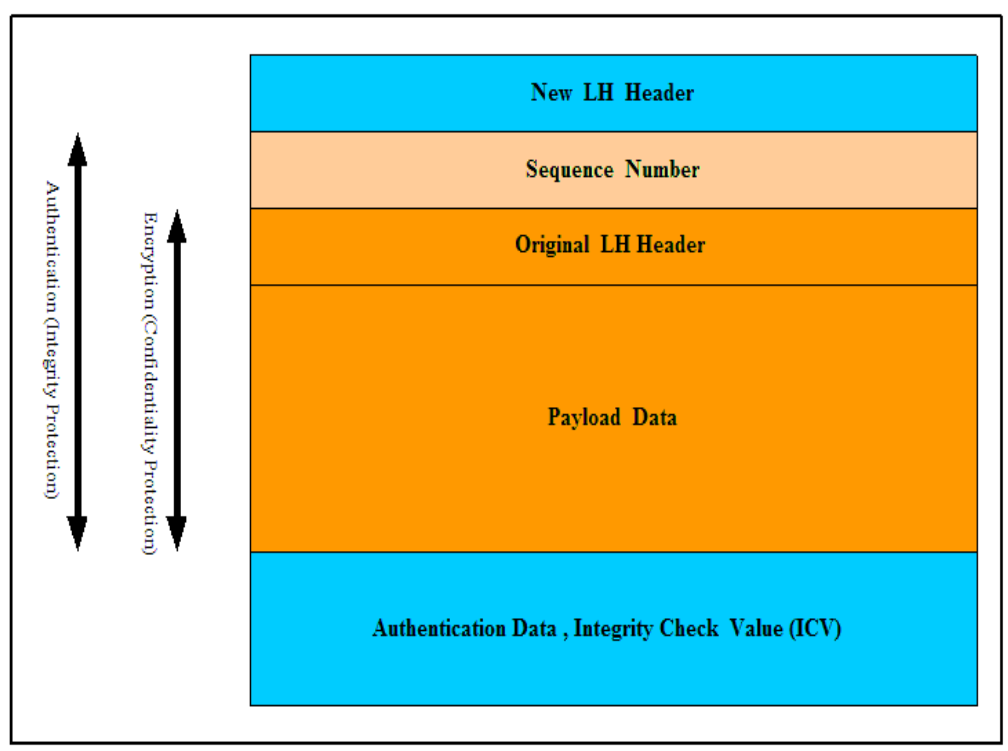

Figure 6. Protocol Structure - DNPSec

The authentication data field containing an Integrity Check Value (ICV) computed over the key sequence number, the original LH header, and the payload data fields. ICV 
provides integrity services and is provided by a specific message authentication algorithm (MAC) such as, HMAC-MD5-96 or HMACSHA-1-96.

\section{Advanced Protocol Structure}

This study propose secure protocol structure of PROFINET/DCP for authentication of packets. It added authentication data field to original PROFINET/DCP protocol. It is similar with DNPSec protocol structure. Integrity Check Value(ICV) is inserted to authentication data field. It will be informs that packet was changed or not.

\begin{tabular}{|c|}
\hline PROFINET/DCPSec \\
\hline FrameID \\
\hline SeviceID \\
\hline Service Type \\
\hline Xid \\
\hline Response Delay \\
\hline DCP Data Length \\
\hline Block \\
\hline Authentication Data \\
\hline
\end{tabular}

Figure 7. PROFINET/DCPSec

\section{Conclusion}

As mentioned above, this study is tried to derive studies how to protect the content of SCADA protocols. That is a secure protocol for PROFINET/DCP similar with DNPSec. It ensure that integrity with authentication field. For this, PROFINET/DCP installed on the SCADA can be protected the attack as like Flooding, Packet Sniffing, and Packet Xid Spoofing. In the future, it will reduce infringement of control system and can import a preemptive prevention. In addition, it is more useful because of it is a result of real traffic analysis with PROFINET/DCP packet.

\section{Acknowledgements}

This work was supported by the IT R\&D program of MSIP/KEIT. [010041560, A development of anomaly detection and a mult-layered response technology to protect an intranet of a control system for the availability of pipeline facilities].

\section{References}

[1] SCADA, http://en.wikipedia.org/wiki/SCADA.

[2] Stuxnet, http://en.wikipedia.org/wiki/Stuxnet.

[3] M. Kiuchi, E. Ohba and Y. Serizawa, "Customizing Control System Intrusion Detection at the Application Layer", The SCADA Security Science Symposium (2009)

[4] H. Hadeli, R. Schierholz, M. Braendle, C. Tuduce and S. Obermeier, "Leveraging Determinism in Industrial Control Systems for Advanced Anomaly Detection and Reliable Security Configuration”, The SCADA Security Science Symposium, (2010).

[5] R. Gula, "Identifying Attacks on Control Systems by Scripting Event Aggregation and Correlation", The SCADA Security Science Symposium, (2007). 
[6] S. Cheung, B. Dutertre, M. Fong, U. Lindqvist, K. Skinner and A. Valdes, "Using Model-based Intrusion Detection for SCADA Networks", (2007).

[7] W. Kim, H. Kim, K. Lee and H. Youm, "Risk Analysis and Monitoring Model of Urban SCADA Network Infrastructure", Journal of The Korea Institute of Information Security \& Cryptology, (2011).

[8] PROFINET, http://en.wikipedia.org/wiki/PROFINET.

[9] PROFINET Manual, http://www.profinet.felser.ch/index.html?dcp_frame.htm.

[10] Form of PROFINET DCP packet, http://www.industrialnetworx.com/forum/profinet/com-flag-not-set.

[11] PROFINET protocol family, http://wiki.wireshark.org/PROFINET.

[12] PROFINET Overview - DCP addressing and subnetting, http://us.profibus.com /docs/pi_white_paper_ profinet_it_en_v1_0.pdf

[13] PROFINET Addressing, PROFINET System Description Technology and Application, 2.5. Addressing

[14] DHCP Addressing, http://support.automation.siemens.com/WW/llisapi.dll?func=cslib. csinfo\&objId=29451913\&nodeid0=18977720\&load=treecontent\&lang=en\&siteid=cseus\&aktprim=0\&objact ion $=$ csview \&extranet $=$ standard \&viewreg $=\mathrm{WW}$.

[15] Constructing and sending DHCP messages, http://www.freesoft.org/CIE/RFC/2131/20.htm.

[16] P. Koh, H. Choi, S. Kim, H. Kwon and H. Kim, "Intrusion Detection Methodology for SCADA system environment based on traffic self-similarity property", Journal of The Korea Institute of Information Security \& Cryptology, (2012).

[17] M. Majdalawieh, F. Parisi-Presicce and D. Wijesekera, "DNPSec: Distributed Network Protocol Version 3 (DNP3) Security Framework", Advances in Computer, Information, and Systems Sciences, and Engineering (2006)

\section{Authors}

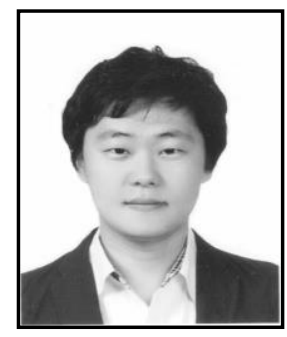

Sangkyo Oh, is now a Master Course in Graduate School of Information Management and Security at Korea University since 2013.

Hyunji Chung, is now a Ph.D Course in Graduate School of Information Management and Security at Korea University since 2012.

Sangjin Lee, received his Ph.D. degree from Korea University. He is now a Professor in Graduate School of Information Security at Korea University and the head of Digital Forensic Research Center in Korea University since 2008. He has published many research papers in international journals and conferences. He has been serving as chairs, program committee members, or organizing committee chair for many domestic conferences and workshops. His research interests include digital forensic, steganography, cryptography and cryptanalysis.

Kyungho Lee, received his Ph.D. degree from Korea University. He is now a Professor in Graduate School of Information Management and Security at Korea University, and leading the Risk management Laboratory in Korea University since 2012. He has a high level of theoretical principles as well as on-site experience. He was a former CISO in NHN corporation, and now he takes as the CEO of SecuBase corporation. His research interests include information security management system(ISMS), risk management, information security consulting, privacy policy, and privacy impact assessment(PIA). 
International Journal of Security and Its Applications Vol.8, No.2 (2014) 